\title{
How to develop a more accurate risk prediction model when there are few events
}

In this Research Methods and Reporting paper (BMJ 2015;351:h3868, doi:10.1136/bmj.h3868), the section "Application of penalised regression" has a few text errors from the third paragraph onwards. A male patient should be considered rather than a female patient, and the risk score is -1.504 rather than -1.714 . The text should therefore read as follows:

"Consider, for example, a male patient aged 20.5 years and with $1.7 \mathrm{~m}^{2} \mathrm{BSA}$, who had a $31 \mathrm{~mm}$ mitral valve manufactured after 1981 from a batch without fractured implants. Using the estimated coefficients from standard regression (table), the risk score for this patient is calculated by the following formula:

Risk score $=-7.8$ (intercept $)+(-0.24 \times 0($ male sex $))+$ $(-0.052 \times 20.5($ age; years $))+\left(1.98 \times 1.7\left(\mathrm{BSA} ; \mathrm{m}^{2}\right)\right)+$ $(2.62 \times 1($ mitral size $31 \mathrm{~mm}))+(0.589 \times 0($ no fracture $))+$ $(1.38 \times 1($ date of manufacture after 1981) $)=-1.504$.

Therefore, the predicted risk of mechanical failure is: $\exp (-1.504) \div(1+\exp (-1.504))=18 \%$ (average risk is $1.8 \%)$. When the estimated coefficients from ridge and lasso are used instead, the predicted risks are less extreme: $12 \%$ and $15 \%$, respectively." 